\title{
Landscape Image Filtering via Aesthetic Inference
}

\author{
Mahsa Ghane ${ }^{1}$, Asadollah Shahbahrami ${ }^{2}$ \\ ${ }^{1}$ Department of Information Technology The University of Guilan, Rasht, Iran \\ ${ }^{2}$ Department of Computer Engineering The University of Guilan, Rasht, Iran
}

\begin{abstract}
Today's because of increasing photography facilities, produce a high number of images. Hence, we need devices and applications for better images search and stronger management of them based on qualities or quantity of aesthetic. These help us to create digital image albums and auto management of contents according to aesthetic concept. In this paper first, we want to introduce the general features in aesthetics in different scopes of photography such as portrait, macros, news, sports, animals and etc. Second, trying to determine the particular influence of each feature on different scopes of photography because aesthetic features are presented in photography are very general. Next, among of these provided features, the top six features that specifically have the highest influence on the landscape images are proposed. Accuracy verification of these features was shown by comparing images from a standard dataset. Supposing the ranking of aesthetic in the dataset from 1 to 7,1 (very ugly)and 7(very beautiful), it is observed that proposed features was influence due to their ranking in dataset were more than 5 and none of them had ranking scores with less than 5 in the standard dataset..
\end{abstract}

Keywords: - Aesthetics Rating, Datasets, Inference, Digital photography

\section{INTRODUCTION}

Nowadays digital cameras have been improved and has added many capabilities such as increase memories size and speed up cameras for a quick shot without delay, and also fast review the recorded photos. All of these features will enable photographs to record a lot of different shots of a landscape with different compositions, diaphragms, speeds and different optical views. Then in the better time, they can compare all of these images and select the best image of a landscape. But on the other hand, in many time these increased facilities make difficult to select good photos by photographers. For example, if increase the number of shots, the photographer can easily record more than 1000 photos at the end of a working day. On average 20 photos of these, related to environment of one landscape. In this situation, select an image as a representative of the photos which observe highest principles of aesthetics like composition rules, the rule of thirds, depth of field, exposure of light is may be hard and boring work.

In this paper we consider the application of aesthetic inference for images management in real world. Immense popularity of photo-sharing communities (e.g., Flickr, Photobucket, Photo.net) and social-networking platforms (e.g., Facebook and Myspace) caused aesthetic inference to be necessary for introduce new capabilities to manage medias. So that in turn may be competitive in these crowded markets. In the case of visual media management, areas such as ontent-based image classi_cation and retrieval [7], automatic annotation [8,9], and image watermarking [10] for rights management have been extensively studied. In this age of digital photo increasing, it is critical to continuously develop intelligent systems for automatic image content analysis. Recent research has shown that measure of visual quality is possible and researchers relatively have been able to recognize aesthetic in successfully manner.

Some features that have been accepted results are in the following: 1. Rule of Thirds: A very popular rule of thumb in photography is the Rule of Thirds. The rule of thirds is a simplified version of the golden mean or golden section $[1,2,6,16,18]$. 2 . The Golden triangle rule: it is another form of golden mean where a diagonal line divides the image, corner to corner [1,19]. 3. Low Depth of Field: it means camera will focus on subject and the background is out of focus so it will be record more blur. In this situation subject seem more prominent $[1,4,6,12,17,18]$. 4. Exposure of light: light exposure can often be a good discriminant between high and low quality photographs $[2,6,16] .5$. Colorfulness: complementary and multi color are some features which is very effective on aesthetics of images $[2,5,6,16,17,18]$. 6. Saturation and hue : Pure colors in a image tend to be more appealing than impure ones $[2,6]$. 7. Familiarity measure: the same images guess using color, texture and shape information of segmented region automatically $[6,11]$. 8. Wavelet-based Texture: Graininess or smoothness in a image can be indicate the presence/absence and nature of texture within the image [3,6]. 9.Aspect ratio: It is well-known that $4: 3$ and $16: 9$ aspect ratios, which approximate the 'golden ratio' $[6,18]$.

The challenge of recognize aesthetic features show as a chart in section 2 . Then introduce the recommended features for landscape images in section 3. In section 4, presented experimental results and efficiency/inefficiency of them in landscape scope and section 5 is conclusion. 


\section{SEMANTIC AND AESTHETIC GAPS}

The aesthetic gap means the people have different understanding of aesthetic and make different ranks and categories to one image. On the other hand, For simulation of aesthetic in machine we need only one rank.

The semantic gap is non-compliance of interpretations. The current technology of computer are incapable to identify and understand the true conceptual of aesthetics of an image. Semantic and aesthetic gaps shown in figure 1 that demonstrate people have different sense of conceptual, aesthetic and categories of a photo, while we try to make all of these comments to a rank in the machine.

Despite the aesthetic and the semantic gaps between machine and human perception, we introduce the proved features and recommend six features for aesthetics in landscape images. Then, we analyse them with statistical results. Obviously, a definitive answer can't be reached with these mismatch of aesthetic perception however, our recommended features provided an acceptable answers [24].

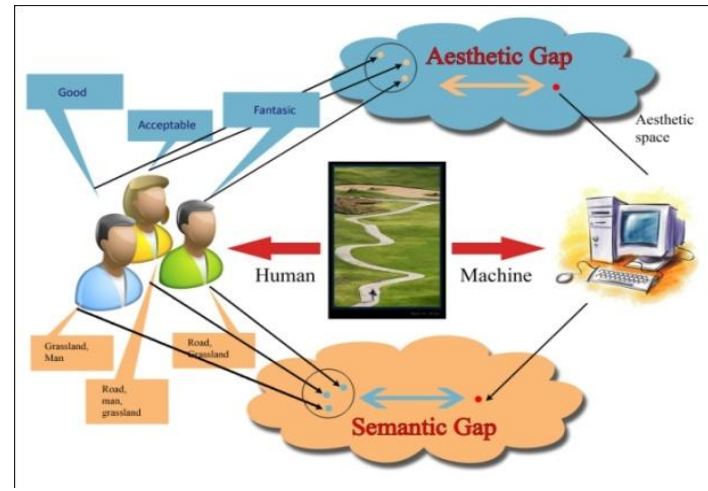

Fig 1. Mismatch between people and machine for aesthetic and semantic perception of one image.

\section{INVESTIGATION THE PROPOSED FEATURES FOR LANDSCAPE IMAGES}

In this section of paper we investigate the "aesthetic features" and "photo composition" for each image of landscape to realizing of a beautiful image. To get the features we have extracted them from images. In fact, feature extraction means extract the primary concept of images. This helps reduce the complexity of image effects and we can do a better measure of an image based on some of the more important factors [2,6]. Photography has many rules for combination in a beautiful image. Some of them have been formulated to recognize aesthetic. During the past century, many artists have used these rules, hence they called "universal composition rules". It means scientific principal and rule for drew and design $[1,6]$.

Landscape images can be included different subjects like: oceans, forests, deserts, mountains, poles, farms, beaches, snow landscapes, lakes, rivers, sunrise and sunset, grass, rocks, roads, fog scenes, waterfalls, swamped, islands, clouds and all variety of them can be record in different seasons. So, there is a wide range of images and we want to introduce features that are priority of aesthetic in all of images. The different classes of images need different features for being good. For example, low depth of field (DoF) is one of the considerable features for portrait or macro images. But this feature is not good for landscape images [4]. According to this view, we recommended six features that are most important in recognizing of aesthetic in landscape images.

The features that we recommend in this paper are: 1.The rule of thirds, 2.Golden triangle rule, 3.Symmetry, 4. Exposure of light, 5. Colorfulness , 6. Aspect ratio. We extract features separately For every downloaded image and use of these symbols : RGB images are transformed to HSV color space. Twodimensional matrices IV 'IS 'IH produced to each dimension of $\mathrm{x}$, y. without meaningful features, it is difficult to achievement meaningful results. In the following referring to the mentioned features as "candidate features".

\section{A. The rule of thirds}

A very popular rule of thumb in photography is the Rule of Thirds. It is the composition rule in photography. This rule states that important compositional elements of the photograph should be situated in one of the four possible intersections -also called power points- of the lines that divide the image into nine equal rectangles, as seen in Fig. 2 [1,2,6,16,18]. The rule can be considered as a sloppy approximation to the 'golden ratio, $\left(\ell=\frac{1+\sqrt{5}}{2} \approx 1.618\right.$ main object stretch from an intersection up to the center of the image. Based on these observations, we calculated the average hue as

$$
f_{1}=\frac{9}{X Y} \sum_{x=X / 3}^{2 X / 3} \sum_{y=Y / 3}^{2 Y / 3} I_{H}(x, y)
$$


with $\mathrm{f} 2$ and $\mathrm{f} 3$ being similarly computed for Is and Iv respectively [6]. A sample of observing this feature is shown in fig.5.a.

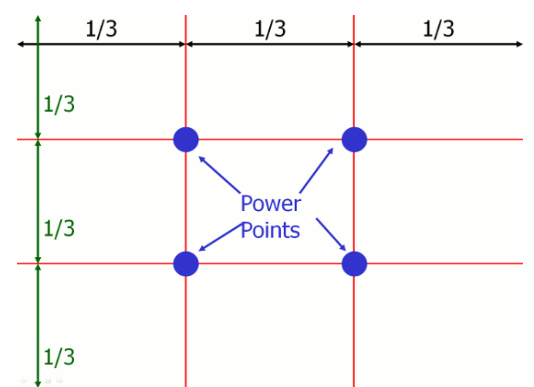

Fig. 2. explanation of The rule of thirds.

\section{B. Golden triangle rule}

The golden triangle rule is a special case of the golden mean, where a diagonal line divides the image, corner to corner. It is the composition rule in photography ( see fig. 5.b)

for calculating of this feature, create three diagonal guides by marking the vertical edges of your screen 1/6th of the height from the top and 1/6th from the bottom. The same way mark the horizontal edges $1 / 6$ th of the width from the left and 1/6th from the right. Connect the upper left horizontal mark with the lower right vertical mark, the upper left vertical mark with the lower right horizontal mark, and the upper left corner with the lower right corner. You may also use the opposite corner combination if you want the oppsite diagonal lines. By placing natural elements that form a line along these diagonal guides makes the picture more pleasing and dynamic.

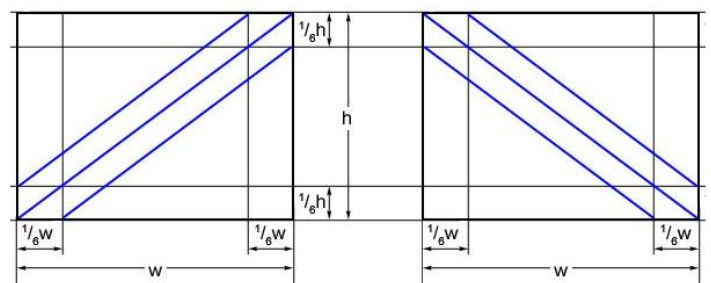

Fig. 3. guideline for recognizing of golden triangle rule

\section{Exposure of light}

Too much exposure (leading to brighter shots) often yields lower quality photos. On the other hand, those that are too dark are often also not interesting. Thus light exposure can often be a good discriminant between high and low quality photos [2,5,6]. An example of this image is shown in fig. 5.c. The average pixel intensity is used to characterize the use of light.

$$
f_{4}=\frac{1}{X Y} \sum_{x=0}^{X-1} \sum_{y=0}^{Y-1} I_{V}(x, y)
$$

\section{Colorfulness}

Colorfulness of images and the complementary color can affect the appearance of aesthetic ( See figure 5.d). We use a fast and robust method to compute relative color distribution, distinguishing multi-colored images from monochromatic, sepia or simply lowcontrast images. We use the Earth Mover's Distance (EMD) [21], which is ameasure of similarity between any two weighted distributions. We divide the RGB color space into 64 cubic blocks with four equal partitions along each dimension, taking each such cube as a sample point. Distribution D1 is generated as the color distribution of a hypothetical image such that for each of 64 sample points, the frequency is $1 / 64$. Distribution D2 is computed from the given image by finding the frequency of occurrence of color within each of the 64 cubes.

The EMD measure requires that the pairwise distance between sampling points in the two distributions be supplied. Since the sampling points in both of them are identical, we compute the pairwise Euclidean distances between the geometric centers $\mathrm{C}_{\mathrm{i}}$ of each cube $\mathrm{i}$, after conversion to LUV space. Thus the colorfulness measure f5 is computed as follows:

$$
f_{5}=e m d\left(D_{1}, D_{2},\{d(a, b) \mid 0 \leq a, b \leq 63\}\right)
$$


where

$$
d(a, b)=\left\|\operatorname{rgb} 2 L U V\left(C_{a}\right)-\operatorname{rg} b 2 L U V\left(C_{b}\right)\right\|
$$

The distribution D1 can be explained as the ideal color distribution of a

'colorful' image. How similar the color distribution of an arbitrary image is to this one is a approximate measure of how colorful that image is. Examples of images producing high and low values of f5 are shown in Fig. 4 [6].
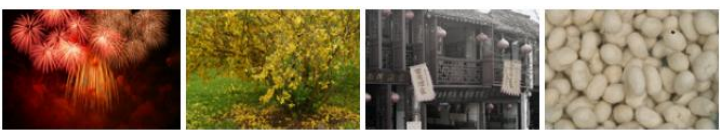

Fig. 4. The proposed colorfulness measure. The two photos on the left have high values while the two on the right have low values.

\section{E. Symmetry}

In the published literature, referred to "symmetry" is one of the main criteria of art aesthetic judgment[14]. In art, symmetry means the equilibrium (the forms, colors, lines, light, etc.) from left to right or top to bottom and overall it is a part of aesthetic quality of art [13]. There are two types of symmetry detection: bilateral Symmetry and rotational Symmetry [22]. bilateral symmetry seen frequency in the world and appears to be associated with different areas. In nature it is commonly seen in living creatures such as butterflies, in still life like flowers, butterfly, birds and etc [23].

bilateral Symmetry can be achieved by making a series of mirror features descriptor and matching them with the against features. Our method is based on matching pairs of symmetric points. A set of feature points $p_{i}$ are determined by SIFT method, which detects distinctive points with excellent repeatability. The point vector $p_{i}=\left(x_{i}, y_{i}, \phi_{i}, s_{i}\right)$ assigned to each feature point describes its location, orientation and (optionally) scale. Scale need only be determined if a scale-invariant feature detection method is used. Orientation, although must be determined as it is main to the evaluation of symmetry. ${ }^{{ }^{i}}$ Show orientation and ${ }{ }_{i}$ demonstrate size

of $x_{i}, y_{i}$ [22]. An example of this image is shown in fig. 5.e.

\section{F. Aspect Ratio} [6]:

The aspect ratio of an image has a good chance of affecting the image ratings. the aspect ratio feature is

$$
f_{6}=\frac{X}{Y}
$$

An example of this image is shown in fig. 5.f. Some of them are features that are referred in the books of landscape photography skills $[16,17,18]$. Furthermore, the rule of thirds, exposure of light, colorfulness and aspect ratio introduced in [6] as 15 top features from 56 cases for aesthetics. It is shown In figure 6 the images that hasn't got the proposed features, so their scores were low.

\section{A. Data set}

\section{Aesthetic eVAluation Results}

In this paper has been used of an standard data set of images in [6]. This data set produced by Photo.net website [15] which provided a platform for photography enthusiasts and photographer can share their pictures and receive a score based on aesthetics and quality of their photo between 1(very ugly) and 7(very beautiful).

We collected 200 images that each one at least rating by two users. 50 images were relate to landscape from this collection that proposed features was performed on these images. 9 cases were presented in this paper as examples.

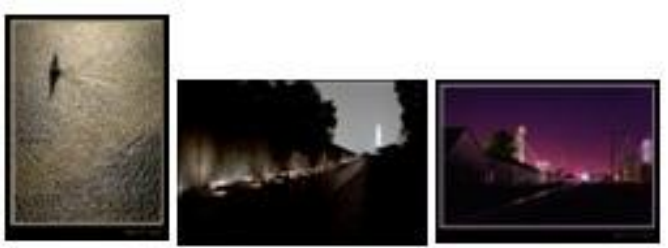


(a)

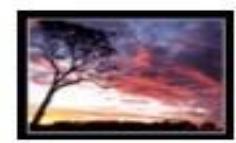

(d) (b)

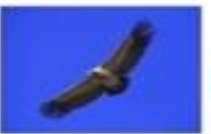

(E) (c)

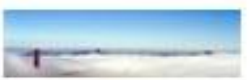

(f)

Fig 5. a) The rule of thirds, b) golden triangle rule, c) exposure of light, d) colorfulness, e) symmetry, f) aspect

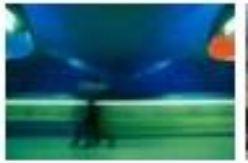

(b)

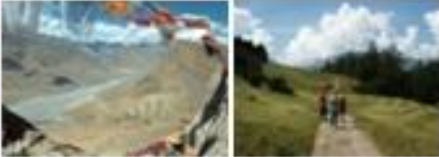

(b) (c)

Fig 6. These are samples of images that hasn't got proposed features and their ranking in data set are low or

\section{B. Evaluation criteria} average.

In this paper, evaluation criteria is subjective. It mean each image ranking in this data set is based on rating and comment of people and the average score has been introduced as aesthetic ranking of images in data set. Image ranking is between 1 to 7 that each mean is in table 1 .

Table 1. Aesthetic concept of images ranking between 1 to 7 in data set.

\begin{tabular}{|c|c|}
\hline $\begin{array}{c}\text { Aesthetic } \\
\text { concept }\end{array}$ & $\begin{array}{c}\text { Image } \\
\text { ranking }\end{array}$ \\
\hline Very ugly & 1 \\
\hline ugly & 2 \\
\hline bad & 3 \\
\hline average & 4 \\
\hline good & 5 \\
\hline beautiful & 6 \\
\hline Very beautiful & 7 \\
\hline
\end{tabular}

\section{Experimental result}

Numerical rating of data set and numerical rating of an expert person (professional photographer) presented in a table together for comparison. These numbers are close to each other, indeed confirmed the expert person opinion in determining aesthetic evaluation. Figure 7 shows Numerical rating of data set and numerical rating of the expert person in comparison manner.

In addition, we want a professional photographer to select some landscape images from date set which observe our proposed features and he determined presence/ absence of features in each of images. Then extract the rating of their images in data set. These results is shown in table 2.

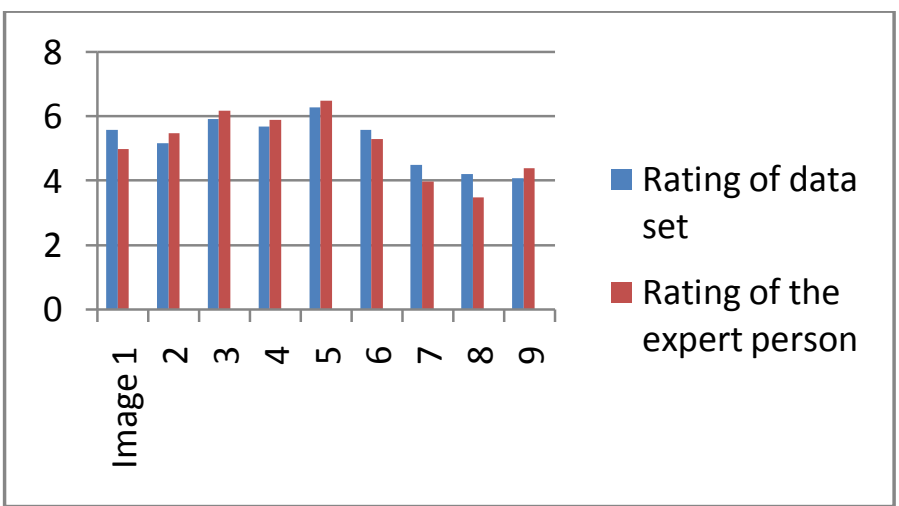

Fig. 7. Similarity ranking chart of rating of data set and rating of the expert person. 
Table 2. The results of features investigation in some of data set images with their ranking.

\begin{tabular}{|c|c|c|c|c|c|c|c|}
\hline & $\begin{array}{c}\text { The } \\
\text { rule } \\
\text { of } \\
\text { thirds }\end{array}$ & $\begin{array}{c}\text { golden } \\
\text { triangle } \\
\text { rule }\end{array}$ & $\begin{array}{c}\text { exposure } \\
\text { of light }\end{array}$ & colorfulness & symmetry & $\begin{array}{c}\text { aspect } \\
\text { ratio }\end{array}$ & Ranking \\
\hline 5.a & $\mathrm{Y}$ & $\mathrm{N}$ & $\mathrm{N}$ & $\mathrm{N}$ & $\mathrm{N}$ & $\mathrm{N}$ & $\mathbf{5 . 5 8}$ \\
\hline 5.b & $\mathrm{N}$ & $\mathrm{Y}$ & $\mathrm{Y}$ & $\mathrm{N}$ & $\mathrm{N}$ & $\mathrm{N}$ & $\mathbf{5 . 1 7}$ \\
\hline 5.c & $\mathrm{N}$ & $\mathrm{N}$ & $\mathrm{Y}$ & $\mathrm{Y}$ & $\mathrm{N}$ & $\mathrm{N}$ & $\mathbf{5 . 9 4}$ \\
\hline 5.d & $\mathrm{N}$ & $\mathrm{N}$ & $\mathrm{Y}$ & $\mathrm{Y}$ & $\mathrm{N}$ & $\mathrm{N}$ & $\mathbf{5 . 7}$ \\
\hline 5.e & $\mathrm{N}$ & $\mathrm{Y}$ & $\mathrm{N}$ & $\mathrm{N}$ & $\mathrm{Y}$ & $\mathrm{N}$ & $\mathbf{6 . 2 9}$ \\
\hline 5.f & $\mathrm{N}$ & $\mathrm{N}$ & $\mathrm{Y}$ & $\mathrm{N}$ & $\mathrm{N}$ & $\mathrm{Y}$ & $\mathbf{5 . 5 8}$ \\
\hline 6.a & $\mathrm{N}$ & $\mathrm{N}$ & $\mathrm{N}$ & $\mathrm{N}$ & $\mathrm{N}$ & $\mathrm{N}$ & $\mathbf{4 . 5}$ \\
\hline 6.b & $\mathrm{N}$ & $\mathrm{N}$ & $\mathrm{N}$ & $\mathrm{N}$ & $\mathrm{N}$ & $\mathrm{N}$ & $\mathbf{4 . 2 1}$ \\
\hline 6.c & $\mathrm{N}$ & $\mathrm{N}$ & $\mathrm{N}$ & $\mathrm{N}$ & $\mathrm{N}$ & $\mathrm{N}$ & $\mathbf{4 . 1}$ \\
\hline
\end{tabular}

\section{Conclusion}

Due to the growing of private and public photograph and also increase demand for new management in this region to automatic image processing, it is important to define the features that have significant relations with aesthetics subjective criteria. In this paper, we investigate and determine some features that have meaningful relations with aesthetic subjective of landscape photography images. In order to better recognizing of some aesthetic features, we use subjective criteria based on results of Photo.net data set. six proposed features of landscape images shown they are right choice for ranking of most beautiful landscape pictures. Our future work is aesthetic simulation for determine automatic image processing with using proposed features.

\section{REFERENCES}

[1] P. Obrador, L. S. Hackenberg and N. Oliver, "The Role of Image Composition in Image Aesthetics", Proc. IEEE Int. Conf. on Image Processing,pp. 3185-3188. 2010

[2] Y. Wu, C. Bauckhage and C. Thurau, "The Good, the Bad, and the Ugly: Predicting Aesthetic Image Labels", Proc. IEEE Int. Conf on Pattern Recognition, pp. 1586-1589. 2010

[3] M. Desnoyer and D. Wettergreen, "Aesthetic Image Classification for Autonomous Agents", Proc. IEEE Int. Conf. on Pattern Recognition, pp. 3452-3455. 2010

[4] L. K. Wong and K. L. Low, "Saliency-Enhanced Image Aesthetics Class Prediction", Proc. IEEE Int. Conf., pp. 997-1000. 2009

[5] W. Jiang, A. C. Loui, and C. D. Cerosaletti, "Automatic Aesthetic Value Assessment in Photographic Images", Proc. IEEE Int. Conf. on Multimedia and Expo, pp 920-925. 2010

[6] R. Datta, D. Joshi, J. Li, and J. Z. Wang, "Studying Aesthetics in Photographic Images using a Computational Approach," Lecture Notes in Computer Science, pp. 288. 2006.

[7] A. W. Smeulders, M. Worring, S. Santini, A. Gupta and R. Jain. "Content-based Image Retrieval at the End of the Early Years". IEEE Trans. Pattern Analysis and Machine Intelligence, 1349-1380, 2000.

[8] C. Carson, S. Belongie, H. Greenspan, and J. Malik. Blobworld: "Image Segmentation using Expectation-Maximization and Its Application to Image Querying". IEEE Trans., 2002.

[9] J. Li and J. Z. Wang. “Automatic Linguistic Indexing of Pictures by a Statistical Modeling Approach”. IEEE Trans. Pattern Analysis and Machine Intelligence, 2003.

[10] I.J.Cox,J.Kilian, F.T.Leighton, and T.Shamoon."Secure Spread Spectrum Watermarking for Multimedia". IEEE Trans., 1997.

[11] J. Z. Wang, J. Li, and G. Wiederhold, "SIMPLIcity: Semantics-Sensitive Integrated Matching for Picture Libraries," IEEE Trans. on Pattern Analysis and Machine Intelli., pp.947-963. 2001

[12] Z. Dai and Y. Wu "Where Are Focused Places of a Photo?", Springer-Verlag Berlin Heidelberg, pp. 73-83. 2007

[13] T. Jacobsen, R.I. Schubotz., and L.Y Hofel, "Brain Correlates of Aesthetic Judgment of Beauty", Neuro Image 2006.

[14] D. W. Zaidel, M. Hessamian, "Asymmetry and Symmetry in the Beauty of Human Faces", Symmetry Journal, 2010.

[15] Photo.net, http://photo.net.

[16] Prakel , "BasicsPhotography:Composition",AV Publishing 2006.

[17] C. Kahn," Essential Skills for Nature Photography”, Amherst Media, Inc 1999.

[18] R. D. Zakia and D. Page, "Photographic Composition: A Visual Guide", Book 2010

[19] L. Yao, P. Suryanarayan, M. Qiao, J. Z. Wang, and J. Li, "OSCAR: On-Site Composition and Aesthetics Feedback through Exemplars for Photographers", The Pennsylvania State University, 2011.

[20] http://www.picture-thoughts.com/photography/compos-ition/angle/

[21] Y. Rubner, C. Tomasi, and L. J. Guibas, “The Earth Mover's Distance as a Metric for Image Retrieval,” Int'1. J, 2000.

[22] G. H. Loy and J. O. Eklundh "Detecting Symmetry and Symmetric Constellations of Features" Computational Vision and Active Perception Laboratory, 2006.

[23] M. Chertok and Y. Keller "A Graph Matching Approach to Symmetry Detection and Analysis" Symmetry Journal 2011.

[24] Ritendra Datta, "Semantics And Aesthetics Inference For mageSearch: Statistical Learning Approaches", Submitted in Partial Fulfllmentof the Requirements for the Degree ofDoctor of Philosophy, 2009 